\title{
Geometrical parameters measurement of surface functionalized micromachined micro-cantilever using optical method
}

\author{
Anil Sudhakar Kurhekar ${ }^{1,2^{*}}$ and Prakash R Apte
}

\begin{abstract}
Micro-cantilever arrays with different dimensions are fabricated by micromachining technique onto silicon $<100>$ substrate. These sputtered gold-coated micro-cantilevers were later surface-functionalized. Scanning electron microscopy (SEM), atomic force microscopy (AFM), and optical diffraction with laser source are employed to characterize the morphology and image measurement of the micro-cantilever arrays, respectively. The spatial resolution produced in the proposed image measurement method is approaching $1 \mu \mathrm{m}$, and the repeatable precision is confirmable in nanometers. Compared with conventional AFM and SEM measurement techniques, the proposed method has demonstrated sufficient flexibility, repeatability, and reliability. The experimental results have been analyzed and presented in this paper for MEMS micro-cantilevers. The scanning white light interferometrybased, two-point high-resolution optical method is presented for characterizing micro-cantilevers and other MEMS microstructures. In this piece of work, we investigate the microstructure fabrication and image measurement of geometrical parameters such as length, width, and step height of micro-cantilevers fabricated using bulk micromachining technique onto silicon $<100>$ substrate.
\end{abstract}

Keywords: Image measurement, Silicon (100), Micro-cantilevers, Scanning white light interferometry

\section{Background}

Accurate measurement of dimensions of microstructures has recently received much attention because of their potential advantages over conventional atomic force microscopy/scanning electron microscopy (AFM/SEM) techniques [1]. A common method to fabricate the micro-cantilevers is to pattern the deposited continuous film using bulk or surface micromachining technique $[1,2]$. However, these methods are demonstrated perfectly only for submicron micro-cantilever arrays. As the micro-cantilever size decreases to nanometer, interesting behavior may be expected. In particular, reduced microcantilever size results in changes of the domain structure [2] and will affect the characterization of the micro-cantilever. One of the other methods to fabricate the nanometer micro-cantilever array is laser micromachining the deposited material onto a silicon substrate $[2,3]$.

\footnotetext{
* Correspondence: askurhekar@gmail.com

${ }^{1}$ Department of Electrical Engineering, Indian Institute of Technology Bombay, Powai, Mumbai 400708, India

2Datta Meghe College of Engineering, Airoli, Navi Mumbai 400708, India
}

Until now, the conventional image measurement technique for planar microstructural properties of microcantilever on silicon $<100>$ substrates have been studied [4]. However, the applicability of optical methods for microstructure arrays is widely established. In this piece of work, we investigate the microstructure fabrication and image measurement of geometrical parameters such as length, width, and step height of micro-cantilevers fabricated using bulk micromachining technique onto silicon $<100>$ substrate (Table 1 ).

Step height measurement is required in many fields including semiconductors, microcircuitry, and printing. Small steps are often measured using a profilometer by calculating the least-squares straight line through the data and then identifying the areas above and below this as being step and substrate. The step height is calculated using least-squares fit to the equation: $Z=a X+b+h \hat{I}^{\prime}$ where $a, b$, and $h$ are unknowns, and $\hat{I}^{\prime}$ takes the value of +1 in the higher regions and -1 in the lower regions. The unknowns $a$ and $b$ represent the slope and intercept of the line. The step height is calculated as twice the 
Table 1 Dimensions of micro-cantilever: designed and measured using the proposed method

\begin{tabular}{llcc}
\hline Serial number & \multicolumn{3}{l}{ Micro-cantilever } \\
\cline { 2 - 4 } & Dimensions & Design & $\begin{array}{l}\text { Measurement after } \\
\text { micromachining }\end{array}$ \\
\hline 1 & Length & $200 \mu \mathrm{m}$ & $184 \pm 0.01 \mu \mathrm{m}$ \\
\hline 2 & Width & $60 \mu \mathrm{m}$ & $50 \pm 0.01 \mu \mathrm{m}$ \\
\hline 3 & Step height & $200 \mathrm{~nm}$ & $180 \pm 0.01 \mathrm{~nm}$ \\
\hline
\end{tabular}

value of the third unknown, $h$. This approach is fine for samples where the flatness of both the step and substrate are good.

\section{Methods}

Two procedures were used to fabricate micro-cantilever arrays. Firstly, silicon $\langle 100\rangle$ substrates were deposited with silicon dioxide using a thermal oxidization procedure [5]. The oxide deposition rate was about $1.5 \mathrm{~nm} / \mathrm{min}$ and the gas flow rate of $18 \mathrm{sccm}$. After the oxidization and patterning, the residual silicon was removed by an anisotropic etchant, tetramethylammoniumhydroxide (TMAH) + deionized water, at $80^{\circ} \mathrm{C}$ for 45 min [6]. Secondly, micro-cantilever arrays were deposited with chrome-gold layer using radio frequency (rf) magnetron sputtering from a gold target at room temperature. The sputtering chamber was firstly pumped down to 180 mTorr. The deposition of chrome was carried out under Ar atmosphere with about 180 mTorr and the gas flow rate of $18 \mathrm{sccm}$. During the deposition process, the continuous thin film of gold [4] was also deposited onto a silicon substrate under the same condition for the convenience of measuring the film thickness.

The sputtered chrome-gold layer is known to exhibit affinity with thiophenol molecules $[7,8]$. Considering this fact, we have dip-coated the piezo-resistive microcantilevers with $1 \mu \mathrm{mol}$ thiophenol in ethanol solution for $3 \mathrm{~h}$ and then rinsed with ethanol for $2 \mathrm{~min}$. The surface becomes functionalized.

The surface morphology of the micro-cantilever arrays was investigated by the scanning electron microscopy (JEOL 2000, JEOL Ltd., Tokyo, Japan). The grazing incidence laser diffraction, which avoids the effect of substrate to the pattern, was used to study the image measurement of the microstructure. The mechanical properties at the temperature $300 \mathrm{~K}$ were measured by atomic force microscopy.

The image measurement of significant parameters of surface-functionalized micromachined micro-cantilevers such as length, width, and step height were obtained using SEEBREZ ${ }^{\circledR}$ optical multi-sensing system (Quality Control Solutions Inc., Temecula, CA, USA) with laser probe and Taylor-Hobson's Form Talysurf ${ }^{\circledR}$ 3-D surface profiler machine (Taylor Hobson Precision Leicester, England) with 3-D Telemap Gold 3-D profile software. The coni-spherical stylus with the base radius of $2 \mu \mathrm{m}$ was used for the contact-mode measurements.

The coordinate measurements were done with SEEBREZ ${ }^{\circledR}$ optical multi-sensing system with laser probe. This system has an auto focus facility. After the sample was prepared for measurements, the origin of the wafer coordinates was put relatively. Then maximum $z$-coordinate was fixed with a 633-nm laser beam marker so that the maximum step that can be measured is $633 / 4=158.25 \mathrm{~nm}$. The measurement of length and width is simple because the two points placed shall be in-plane. However, for the measurement of step height, since any two points shall not be inplane, we fix one point (maximum $Z$ ) using the marker, and the other point can be placed where the height need to be measured (minimum $Z$ ). The numerical difference between maximum $Z$ and minimum $Z$ of the markers $(\Delta d)$ shall give the step height. The film step height $(\Delta d)$ is directly proportional to the wavelength of the laser light and is inversely proportional to twice the refractive index of the film being etched. Thus, the film step height, using this proposed method, is measured with the relation in Equation 1 [9],

$$
\Delta d=\lambda / 2 \cdot \eta
$$

where $\lambda$ is the wavelength of the laser light and $\eta$ is refractive index of the etched layer ( $\mathrm{Au}$, refractive index $=0.181$ ) [9]. We have measured the length, width, and step height using the proposed method, which is relatively easy and accurate. The $x, y$, and $z$ coordinates of any parts of the structure are measured accurately.

\section{Results and discussions}

Figure 1 shows the proposed two-point-high-resolution and accurate optical method for image measurement of the micro-cantilever array. Figure 2 shows the SEM micrograph of the micro-cantilever array, free-standing in a trapezoidal anisotropically etched microcavity with a thickness of $200 \mathrm{~nm}$. The micro-cantilever has a wider parameter distribution, where the mean parameters deviation is approximately $10 \mathrm{~nm}$. Further analysis of SEM micrograph shows that there is some etch-product reminiscent at the bottom of the trapezoidal etch-pit. However, the sidewalls of the etch-pit are smooth. Figure 3 shows the atomic force microscopy micrograph of the sample. It confirms that the film with micro-cantilevers arrays is free-standing in a trapezoidal microcavity. The close observation of AFM micrograph shows clusters of green Y-shaped tentacles of thiophenol molecule adsorbed on Au surface. Further analysis shows that the 


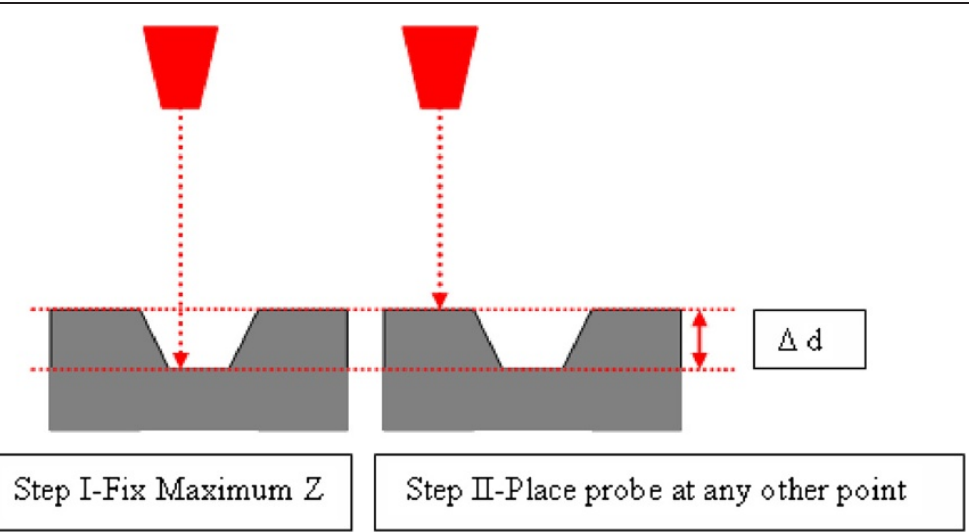

Figure 1 Proposed two-point optical method for etch-depth measurement.

crystallite size distributes is in the range of 5 to $10 \mathrm{~nm}$, calculated with the Scherrer formula [10].

To get the etch-depth information, the micromachined sample was kept on the base of a TaylorHobson's Form Talysurf ${ }^{\mathrm{B}}$ 3-D surface profiler machine. The coni-spherical stylus was reset to its original position after deciding the frame of $x-y$ travel. The machine starts building the etch-profile slowly as the coni-spherical stylus moves over the sample. Threedimensional profile generated using Taylor-Hobson's Talysurf profiler machine of the sample are shown in Figure 4, with the contact mode using coni-spherical stylus moving parallel to the film plane. The image generated using the stylus-based profilometry is in conformity with the scanning electron microscopy. Figures 5 and 6 depict the length and width measurement with SEEBREZ ${ }^{\circledR}$ machine in high accuracy and repeatable precision. The measurements obtained with the proposed optical method are depicted in Table 1.

A two-dimensional profile to recover the depth information is depicted in Figure 7. Etch profile is very important in assessing the etch-uniformity, sidewall smoothness, and etch-depth. Etch-profile also infers the shape, slope, and etch-depth of the microcavity, in which

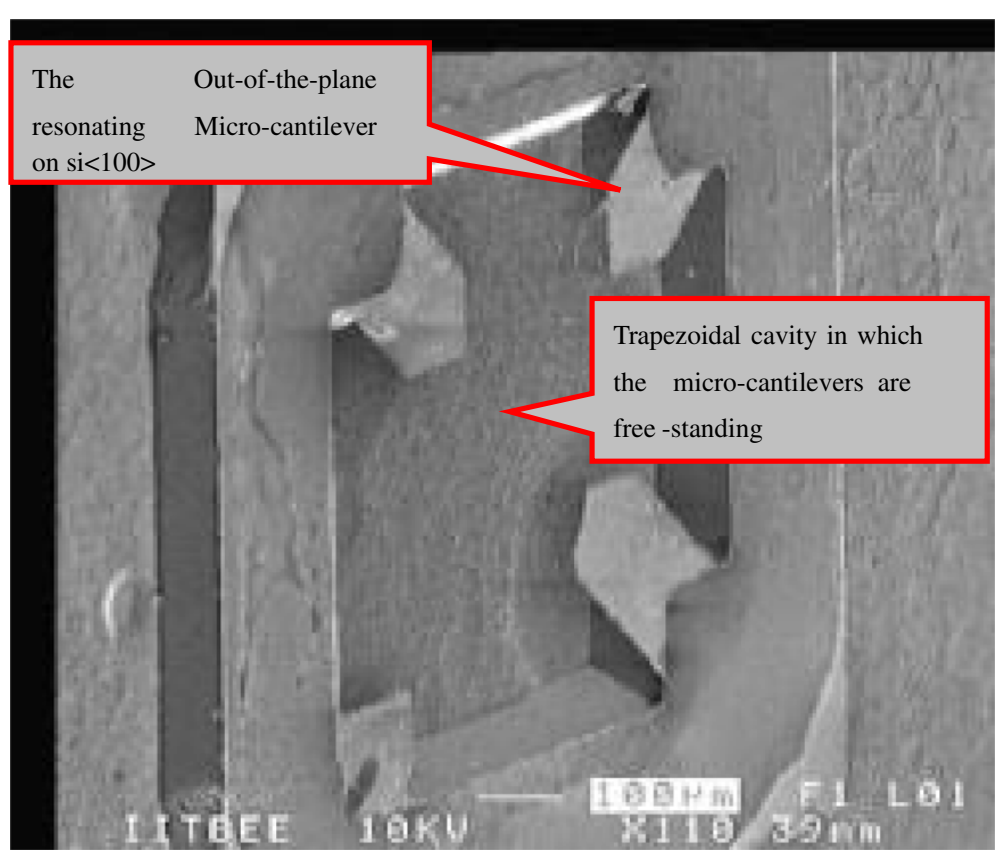

Figure 2 Scanning electron microscopy micrograph of micro-cantilever on silicon $<100>$ surface. 


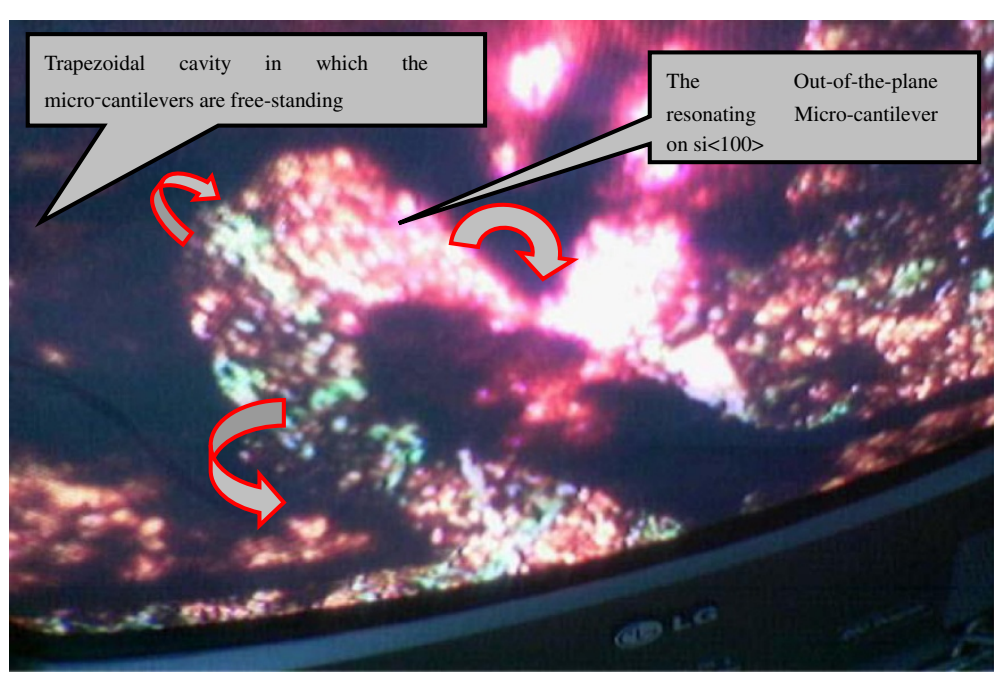

Figure 3 Atomic force microscopy micrograph of surface-functionalized micro-cantilever on silicon $<100>$ surface. The small pointer indicates the out-of-the-plane micromachined micro-cantilever. The wide pointer indicates the trapezoidal microcavity in which the micro-cantilevers are free-standing. The curved arrows indicate the displacements (bending directions) of micro-cantilever due to residual thin-film stress.

the micro-cantilevers are free-standing. It is obvious from the inspection of etch-profile of the trapezoidal microcavity that the sidewalls of this anisotropically etched trapezoidal microcavity are smooth, since its profile is not jagged. However, the bottom of the anisotropically etched microcavity is not smooth, since the profile line is jagged containing hills and dales. The inspection of the etchprofile and scanning electron micrograph confirm that the sidewalls are smooth and at the bottom of the trapezoidal microcavity, there is some etched product reminiscent.

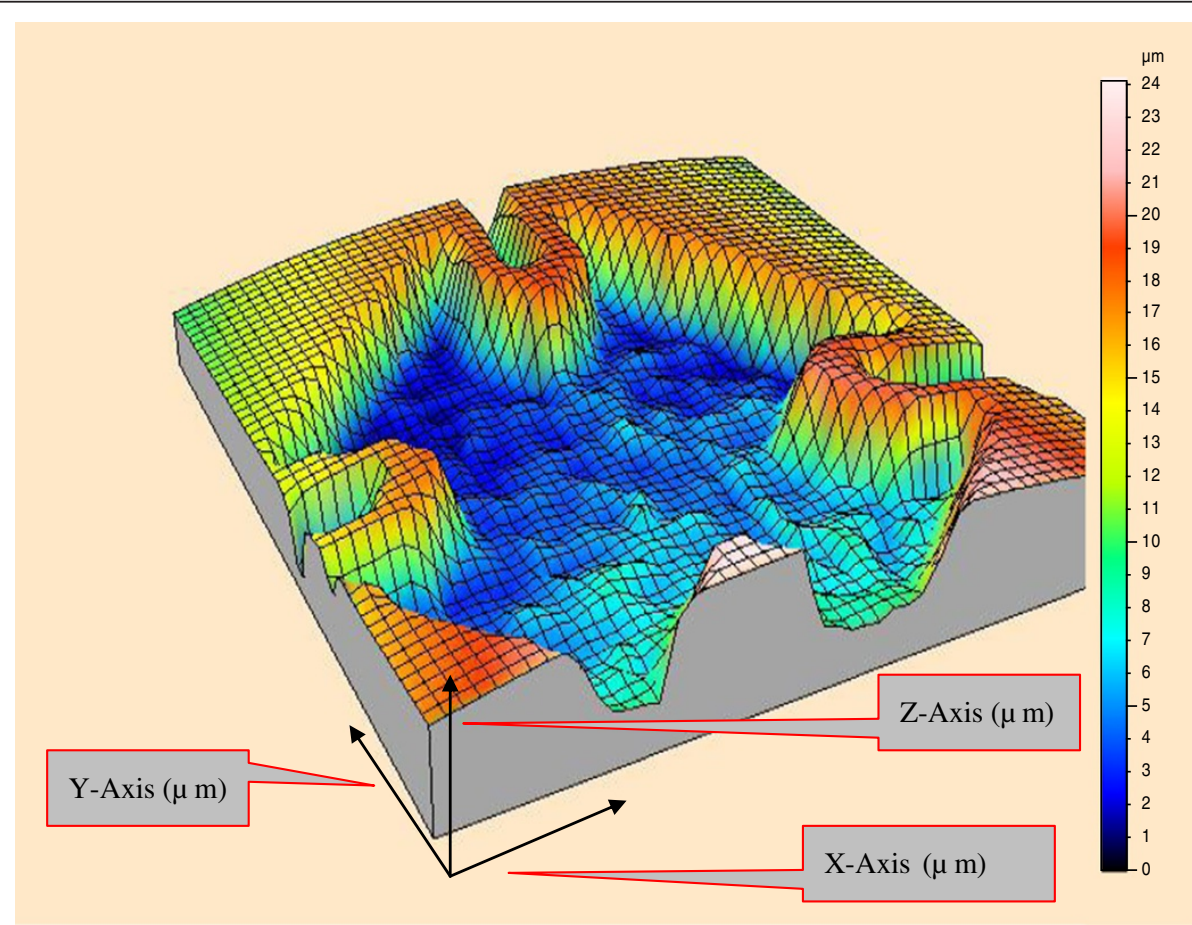

Figure 4 3-D surface profiles (silhouettes) of micro-cantilever the $x-, y$-, and $z$-axes (microns) are shown by the arrows. The origin is placed relatively. 


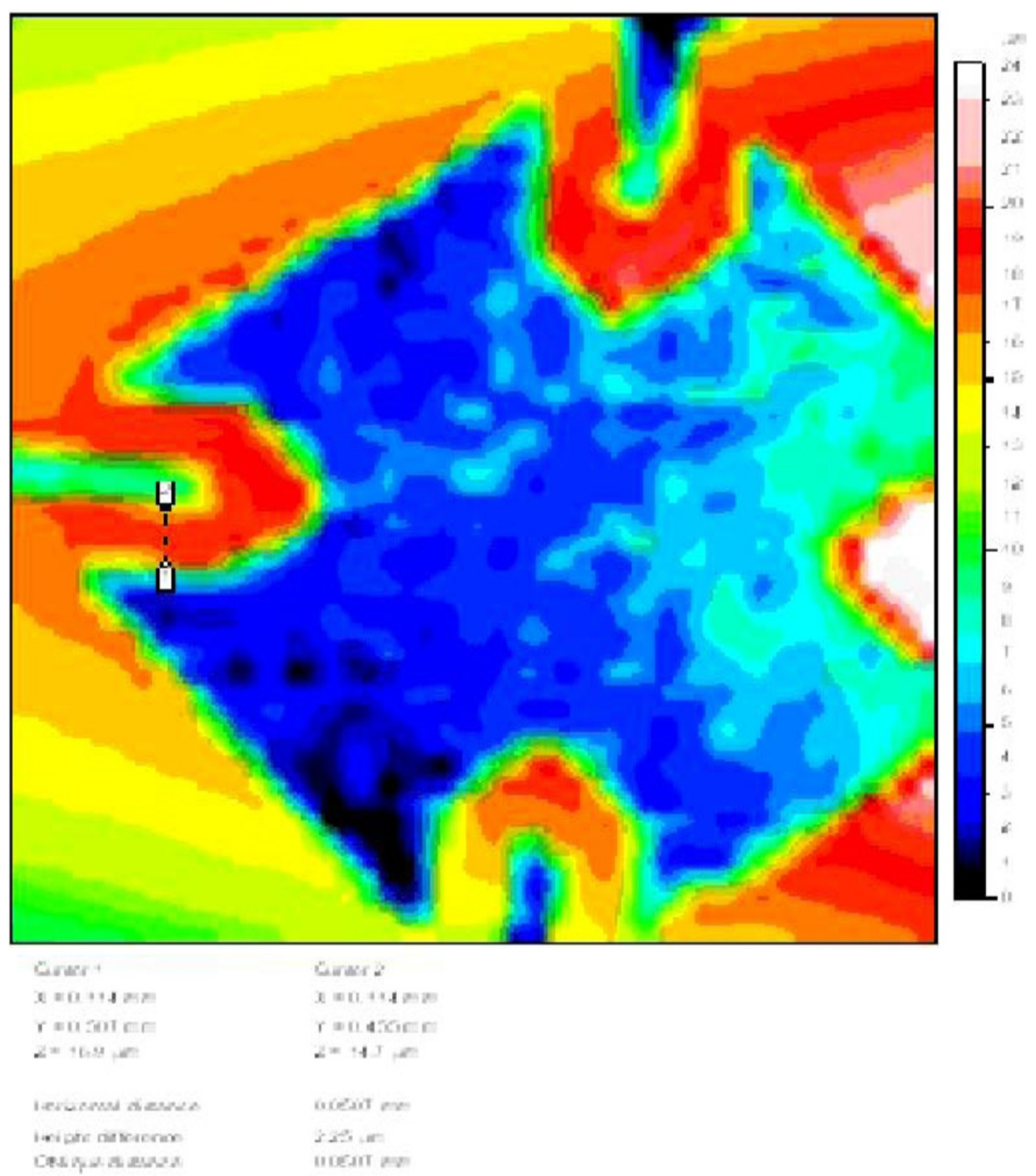

Figure $\mathbf{5}$ Width measurement of micro-cantilever. After placing the origin relatively and fixing the $z$ max, we placed the laser probes at any of the two points (probe points number indicated in square) for device/feature measurements (the original images obtained are having low resolution as is obvious and cannot be further improved).

\section{Conclusions}

In summary, micro-cantilevers array were successfully fabricated on silicon $<100>$ substrate using bulk micromachining technique and deposited with a chrome-gold layer using the rf magnetron sputtering method. These micro-cantilever arrays were surface-functionalized using 1 $\mu \mathrm{mol}$ thiophenol in ethanol solution for $3 \mathrm{~h}$ and after rinsing with ethanol solution for $2 \mathrm{~min}$. The micro-cantilever surface becomes functionalized for mass sensing. The length, width, and step height measurements of microcantilever are obtained with the noncontact method. We propose a high-resolution and accurate two-point optical method for noncontact measurement of step height, which is a novel method.

Where surface tension in the inks or paints causes either a rounding or dimpling of the 'step' and the stresses caused by the curing process can cause distortion in the substrate, the curvature of the substrate might well be sufficient to prevent the use of the simple least-squares line fit. The step and substrate areas are then treated as line segments, which allow the curvature of the substrate to be removed and result in a straightline representation of the substrate. The step heights are calculated from this line in the areas adjacent to each step.

The inspection of the etch-profile and scanning electron micrograph confirm that the sidewalls are smooth and at the bottom of the trapezoidal microcavity, there is some etch product reminiscent. With this substantial conclusion, we propose a high-resolution accurate method for the exact measurement of length, width, and etch-depth of the micromachined micro-cantilever. 


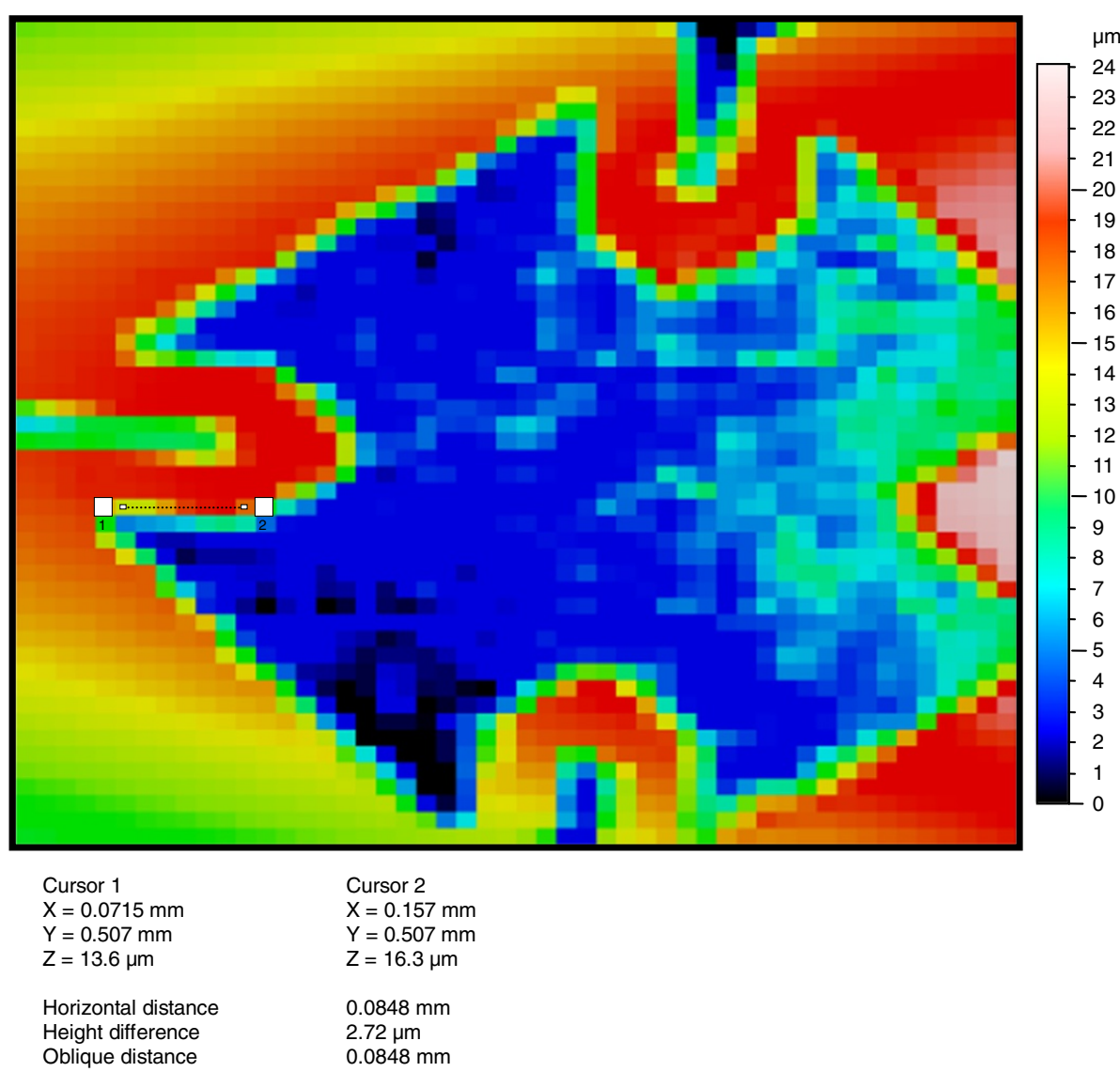

Figure 6 Length measurement of micro-cantilever. (After placing the origin relatively and fixing Zmax, we place the laser probes at any two points (probe points number indicated in square) for device/feature measurements (the original images obtained are having low resolution as is obvious and cannot be further improved).

Further, applicability of this method can be extended for the measurement of significant parameters of other outof-the-plane MEMS structures.

The principal limitation with use of this proposed method is that the minimum probe point separation must be $158.25 \mathrm{~nm}$ and, hence, shall limit the measurement of features less than $158.25 \mathrm{~nm}$. The minimum number of points would reduce the signal-to-noise ratio that may be offset by the ability to average several scans. The rapid scans would also reduce the sensitivity of the instrument to certain environmental effects, such as mechanical drift due to temperature and vibration.

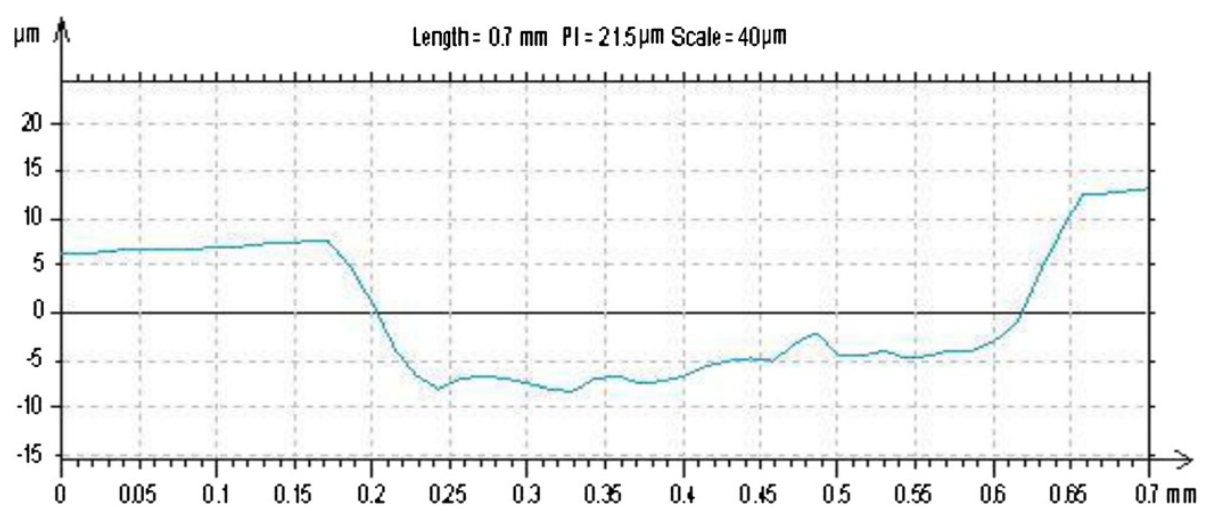

Figure 7 Etch-profile of micro-cantilever. 


\section{Competing interests}

The authors declare that they have no competing interests.

\section{Authors' contributions}

PRA conceived the study. ASK has carried out the design, fabrication and analysis part of this study. Both authors read and approved the final manuscript.

\section{Authors' information}

ASK received his bachelor degree from the Amaravati University in India in 1988 and Master in Engineering degree from Dr. B. A. M. University in India in 1993, both in Electronics Engineering. He is currently pursuing a Ph.D. degree with the Department of Electrical Engineering, Indian Institute of Technology in Bombay, India. His research interest includes MEMS layout, design, simulation and fabrication.

PRA received his bachelor degree from the Indore University of India, in 1968 and the Master of Technology degree from Indian Institute of Technology, Kanpur, India, in 1993, both in electronics engineering. He was conferred Ph.D. by the University of Mumbai in 1988. He is currently a professor in the Department of Electrical Engineering, Indian Institute of Technology in Bombay, India. He was a member of the team that made the first TTL IC in India in 1972. Since then, he has more than 20 years of experience in MOS/Bipolar IC design and fabrication. He has worked at Stanford IC labs for 2 years (1977-1978) as a visiting research associate. To his credit, there are 79 publications in the silicon technology, which includes journal and international conference papers. His current research interest includes MEMS - design, layout, mask-making, fabrication, packaging, testing, and reliability.

\section{Acknowledgements}

The authors acknowledge the Microelectronics Group, Nanoelectronics Centre, Department of Electrical Engineering, Department of Chemistry, Department of Physics, Suman Mashruwala Micromachining Laboratory Department of Mechanical Engineering, Indian Institute of Technology Bombay, Powai, Mumbai and Precision Engineering Division, Bhabha Atomic Research Centre, Trombay, Mumbai, India.

Received: 8 October 2012 Accepted: 8 October 2012

Published: 17 October 2012

\section{References}

1. Apte, PR, Vaishnav, UD, Lokhare, SG, Palkar, VR, Pattalwar, SM: Micromechanical components with novel properties. Proc. of SPIE 3321, 287-297 (1996)

2. Madou, MJ: Fundamentals of Microfabrication: the Science of Miniaturization. CRC, Florida (2002)

3. Wang, $X, L u, P$, Dai, N, Liao, C, Wang, Y, Zheng, Q, Guo, X, Zhang, Q: Femtosecond laser direct fabrication of metallic cantilevers for microcorrosion-fatigue test. J. Micromech. Microeng. 17, 1307 (2007)

4. Sun, Z, Weckenmann, A: Reflective properties of typical microstructures under white light interferometer. Meas Sci Technol 22(08), 103 (2011)

5. Deal, B: The oxidation of silicon in dry oxygen, wet oxygen and steam J Electrochem Soc 10(6), 527-533 (1963)

6. Steinsland, E, Finstad, T, Hanneborg, A: Etch rates of (100), (111) and (110) single-crystal silicon in TMAH measured in situ by laser reflectance interferometer. Sensor Actuat A-Phys 86(1-2), 73-80 (2000)

7. Seelenbinder, JA, Brown, CW, Urish, DW: Self-assembled monolayers of thiophenol on gold as a novel substrate for surface-enhanced infrared absorption. Appl Spectrosc 54(3), 366-370 (2000)

8. Remacle, F, Kryachko, ES: Thiophenol and thiophenol radical and their complexes with gold clusters Au5 and Au6. J Mol Struct 708(1-3), 165-173 (2004). doi:10.1016/j.molstruc.2004.02.056

9. Palik, ED: Handbook of Optical Constants of Solids. Academic, Orlando (1991)

10. Mikrajuddin, A, Khairurrijal, K: Derivation of Scherrer relation using an approach in basic physics course. Jurnal Nanosains \& Nanoteknologi 1(1), 28-32 (2008)

doi:10.1186/2228-5326-2-25

Cite this article as: Kurhekar and Apte: Geometrical parameters measurement of surface functionalized micromachined micro-cantilever using optical method. International Nano Letters 2012 2:25.

\section{Submit your manuscript to a SpringerOpen ${ }^{\circ}$ journal and benefit from:}

- Convenient online submission

- Rigorous peer review

- Immediate publication on acceptance

- Open access: articles freely available online

- High visibility within the field

- Retaining the copyright to your article

Submit your next manuscript at $\boldsymbol{\wedge}$ springeropen.com 